\title{
EFEITO DO NÚMERO DE NEURÔNIOS NA CAMADA OCULTA PARA RELAÇÕES HIPSOMÉTRICAS DE EUCALIPTO USANDO REDES NEURAIS ARTIFICIAIS
}

\author{
EFFECT OF NUMBER OF HIDDEN LAYER NEURONS FOR HEIGHT-DIAMETER \\ RELATIONSHIP OF EUCALYPTUS USING ARTIFICIAL NEURAL NETWORKS \\ Gabriel Fernandes Bueno ${ }^{1}$, Emanuel Arnoni Costa ${ }^{1}$, Amanda Nunes Cristina ${ }^{1}$, \\ Alvaro Augusto Vieira Soares ${ }^{1}$, Rodrigo Otávio Veiga de Miranda ${ }^{1}$, \\ Cristine Tagliapietra Schons ${ }^{2}$ \\ ${ }^{1}$ Universidade Federal de Uberlândia, Monte Carmelo, Minas Gerais, Brasil - \\ gabrielfbueno@outlook.com, emanuelarnonicost@hotmail.com, mandinhanunesousa@gmail.com, \\ alvaroavsoares@gmail.com \& rov_miranda@yahoo.com.br
}

${ }^{2}$ Universidade Federal de Santa Maria, Santa Maria, Rio Grande do Sul, Brasil - cristschons@gmail.com

RESUMO

As redes neurais artificiais têm mostrado performance melhor ou pelo menos similar à dos modelos tradicionais de regressão usados na modelagem florestal. No entanto, deve-se atentar para uma configuração que garanta estimativas acuradas e capacidade de generalização, sendo a quantidade de neurônios na camada oculta uma característica crítica a ser decidida. Assim, o presente estudo buscou encontrar a quantidade ótima de neurônios na camada oculta na modelagem da relação hipsométrica em plantio de Eucalyptus saligna Smith. A base de dados foi subdividida de modo aleatório em $50 \%$ para treinamento e $50 \%$ para validação do modelo, empregando-se a arquitetura de rede neural Multilayer Perceptron (MLP). No processo de modelagem, considerou-se o diâmetro à altura do peito como variável de entrada e a altura como variável de saída. A quantidade de neurônios na camada oculta foi testada com variação entre 1 a 6 . Das seis melhores redes treinadas, aquelas que obtiveram neurônios acima de três na camada oculta tenderam a apresentar overfitting quando aplicados aos dados da validação. Com as diferentes arquiteturas na camada oculta, foi possível obter redes treinadas com coeficiente de correlação $(r)$ variando de 0,61 a 0,73 e raiz quadrada do erro quadrático médio (RQEM) de $\pm 1,28$ a 1,48 $\mathrm{m}$. Foram escolhidas as RNA com três neurônios na camada oculta devido a sua simplicidade e pouca diferença de acurácia quando comparadas às obtidas com maior número. A quantidade de neurônios a serem considerados na camada oculta deve ser definida com atenção para que o fenômeno modelado conserve capacidade de generalização.

PALAVRAS-CHAVE: Altura-Diâmetro, Aprendizado de Máquina, Mensuração Florestal.

\section{ABSTRACT}

Artificial Neural Networks have been shown to perform better or at least similar to the traditional regression models used in forest modeling. However, caution must be taken to choose a configuration the allows for accurate estimates as well as generalization capacity, being the number of neurons in the hidden layer a critical feature to be decided. Therefore, the present study sought to find the optimal number of neurons in the hidden layer for an ANN to model the hipsometric relationship in a Eucalyptus saligna Smith planting. The database was randomly subdivided, $50 \%$ to training and $50 \%$ to model validation, using Multilayer Perceptron (MLP) neural networks algorithm. In the modeling process, the diameter at breast height was considered as input variable and the height as output variable. Quantity variation of hidden layer neurons were tested, ranging from 1 to 6 were tested. The six best trained networks, those that obtained neurons above three in the hidden layer tended to overfitting when applied to the validation data. With the different architectures in the hidden layer it was possible to obtain trained networks with correlation coefficient (r) varying between 0.61 to 0.73 and mean squared error (RQEM) between \pm 1.28 to $-1.48 \mathrm{~m}$. ANN with three neurons in the hidden layer were chosen, in the fact of their simplicity and lower accuracy difference when compared to those obtained with largest number. The quantity of neurons to be considered in the hidden layer must be defined with attention to the model phenomena to conserve the capacity of generalization.

KEYWORDS: Height-Diameter, Machine Learning, Forest Mensuration. 


\section{INTRODUÇÃO}

Nos plantios comerciais é de suma importância seu conhecimento quantitativo, dado que essas informações terão influência no plano de manejo e colheita (CAMPOS et al., 2017), além de possibilitar a realização de análises econômicas do plantio. Em termos gerais, o volume é a variável mais empregada para avaliação quantitativa, obtido por meio do diâmetro e, em muitos casos, da altura da árvore.

A medição de diâmetros pode ser realizada rapidamente e precisamente, utilizando-se sutas ou fitas diamétricas. Contudo, as medições de altura demandam maior tempo e estão sujeitas à maior erro em razão da possível baixa visibilidade da copa (TUAN et al., 2019). Visto que tais fatores aumentam o tempo de execução e custo do inventário, apenas algumas árvores no povoamento são medidas e, por meio destas, modelos que estimam a altura em função do diâmetro são desenvolvidos (SCHMIDT et al., 2011).

O volume pode ser obtido de diferentes maneiras, porém, é comumente estimado por meio de equações volumétricas, tradicionalmente desenvolvidas utilizandose análise de regressão. Na tentativa de obtenção de maior acurácia nas estimativas de variáveis dendrométricas, novas técnicas têm sido comumente testadas, destacandose as Rede Neurais Artificiais (RNA) (VIEIRA et al., 2018). As RNA representam uma alternativa aos métodos tradicionais de modelagem (CAMPOS et al., 2017).

As Redes Neurais Artificiais (RNA) foram desenvolvidas visando simular o sistema nervoso biológico por meio da combinação de várias unidades de processamento simples, chamadas neurônios artificiais, resultando em um sistema extremamente conectado, buscando que o resultado dessa auto-organização e aprendizado seja a "inteligência" (SATO, 1995). A eficiência, adaptabilidade e principalmente a capacidade de resolver problemas torna as RNA uma ferramenta extremamente importante em diversas análises (TKÁČ \& VERNER, 2016).

Alguns estudos vêm demonstrando que as RNA podem ser um método alternativo eficiente nas Ciências Florestais. Como exemplos, as RNA foram aplicadas para obtenção de estimativas de volumes (LEAL, 2015; LACERDA et al., 2017); de biomassa (MONTANO et al., 2017; ÖZÇELIK et al., 2017); de diâmetro à altura do peito (d) a partir de variáveis de sensoriamento remoto ou fatores ambientais (ZHOU et al., 2019); para predições do afilamento do fuste (SCHIKOWSKI et al., 2015; SAKICI \& OZDEMIR, 2018); e, até mesmo, para prever as propriedades físicas e mecânicas de madeira termicamente modificada com base na mudança de cor (NASIR et al., 2019).

Apesar do crescente número de estudos relacionados às RNA na área florestal, investigações relacionadas à definição de uma arquitetura apropriada da rede, como o estabelecimento do número ideal de neurônios na camada oculta, ainda são um grande desafio.

Essas definições podem ser realizadas com base em expressões, como o método de Fletcher-Gloss (SILVA et al., 2010), no entanto podem variar conforme cada aplicação, evidenciando a necessidade de avaliações. Além disso, carecem estudos que avaliem especificamente os efeitos no treino e validação de equações de simples entrada nas RNA.

Definir a quantidade ideal de neurônios na camada oculta é extremamente importante, visto que um número excessivo de neurônios pode acarretar a memorização dos dados de treinamento, processo conhecido como overfitting. De maneira oposta, quando o número de neurônios na camada oculta é pequeno, pode não ser suficiente, processo conhecido como underfitting (BRAGA et al., 2000).

Face o exposto, o presente estudo teve por objetivo aplicar a modelagem hipsométrica usando redes neurais artificiais (RNA) para um plantio de Eucalyptus Saligna. Especificamente, objetivou-se: i) determinar o número ideal de neurônios da camada oculta; ii) treinar cem RNA e avaliar suas precisões e acurácias; iii) selecionar as RNA ideais para os dados usados no treinamento; iv) avaliar a performance da RNA quando testadas nos dados de validação.

\section{MATERIAL E MÉTODOS}

As árvores de Eucalyptus Saligna Smith. usadas no presente estudo são de um plantio florestal de sete anos de idade e espaçamento de 3,0 x 2,0 m, localizado no município de Rosário do Sul, RS. No local, foram obtidos uma frequência de, aproximadamente, 1.025 árvores.ha-1 e área basal de $29,3 \mathrm{~m}^{2}$.ha-1. De acordo com a classificação de Köppen, o clima é do tipo Cfa, subtropical úmido, com precipitação acumulada anual de $1.820 \mathrm{~mm}$ e temperatura média anual de $18,0^{\circ} \mathrm{C}$ (ALVARES et al., 2013).

Um total de 160 árvores compuseram a base de dados utilizada no atual estudo. A variável altura (h) foi mensurada com o hipsômetro Vertex III e o diâmetro à altura de 1,30 $\mathrm{m}$ (d) com uma suta. A base de dados foi subdividida de modo aleatório em $50 \%$ para treinamento das RNA (80 pares de dados) e 50\% reservados para validação (80 pares de dados). O algoritmo de rede neural Multilayer Perceptron (MLP) foi utilizado para construção 
dos modelos, aplicando-se a seguinte arquitetura: 1-n-1. O n significa o número de neurônios da camada oculta, sendo testado a quantidade de 1 a 6 (Figura 1).

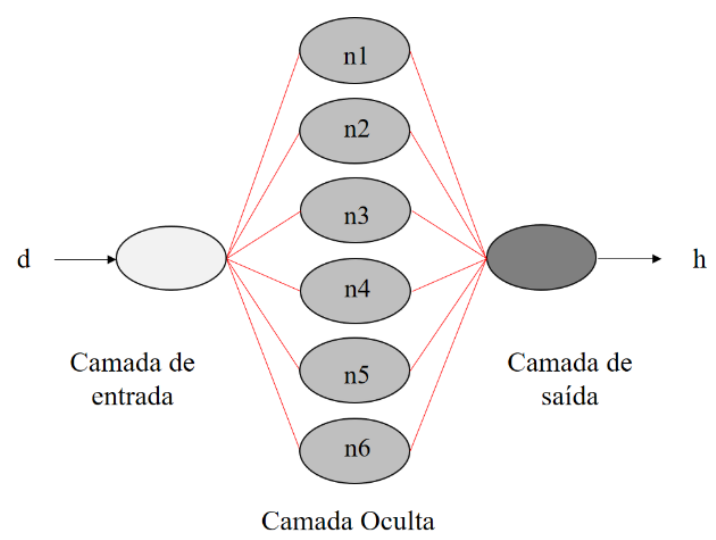

Figura 1. Arquitetura da perceptron de múltiplas camadas testadas para descrever a relação hipsométrica de árvores de eucalipto.

Estudos indicam a possibilidade de optar por configurações simples (BINOTI et al., 2014; LEAL et al., 2015), sendo também usado o método Fletcher-Gloss (SILVA et al., 2010), dado pela expressão 1.

$$
2 . \mathrm{k}^{0,5}+\mathrm{N}_{2} \leq \mathrm{N}_{1} \leq 2 . \mathrm{k}+1
$$

Em que: $\mathrm{k}=$ número de entradas de rede; $\mathrm{N}_{1}=$ o número de neurônios na camada oculta e $\mathrm{N}_{2}=0$ número de neurônios na camada de saída.

No processo de modelagem, para a camada de entrada utilizou-se a variável diâmetro à altura de 1,30 $\mathrm{m}$ (d) e a camada de saída foi a altura total (h) das árvores. $\mathrm{Na}$ camada oculta e na de saída aplicou-se a função de ativação sigmoidal. Um total de 100 RNA foram treinadas para cada arquitetura. A acurácia e precisão das redes foram avaliadas com as seguintes estatísticas: coeficiente de correlação linear entre os valores observados e estimados $(r)$ e a raiz quadrada do erro quadrático médio (RQEM). Os resultados destas estatísticas para cada treino foram analisados graficamente por arquitetura como forma de visualizar a performance dos treinos, conforme utilizado em alguns estudos, como o de Vendruscolo et al. (2015) e Özçelik et al. (2017). Ainda, foi avaliada a distribuição dos resíduos em função do diâmetro observado.

Além disso, para as estatísticas $r$ e RQEM , calculou-se a diferença entre a média das seis melhores redes para cada uma das outras arquiteturas destinadas a avaliação da variação média da performance das diferentes arquiteturas comparadas com a arquitetura mais simples.
As expressões das estatísticas utilizadas para avaliar a acurácia e precisão são definidas por:

i) coeficiente de correlação linear entre as alturas observadas e estimadas $(r)$ :

$$
\mathrm{r}=\frac{\operatorname{Cov}\left(h_{\mathrm{i}}, \hat{h}_{i}\right)}{\sqrt{\mathrm{S}^{2}\left(h_{\mathrm{i}}\right) \mathrm{S}^{2}\left(\hat{h}_{i}\right)}}
$$

ii) raiz quadrada do erro quadrático médio (RQEM):

$$
\text { RQEM }=\sqrt{\mathrm{n}^{-1} \sum_{\mathrm{i}=1}^{\mathrm{n}}\left(h_{\mathrm{i}}-\hat{h}_{i}\right)^{2}}
$$

iii) Resíduos:

$$
\text { Resíduos }=h_{\mathrm{i}}-\hat{h}_{i}
$$

Em que, $h_{\mathrm{i}}$ : altura observada; $\hat{h}_{i}$ : altura estimada; Cov: covariância; $S^{2}$ : variância; $n$ : número de árvores.

iv) delta do coeficiente de correlação $\left(\Delta_{\mathrm{r}}\right)$ :

$$
\Delta_{\mathrm{r}}=\text { Média }(\mathrm{r}) \mathrm{N}[2 \ldots 6]-\text { Média }(\mathrm{r})_{\mathrm{N}[1]}
$$

v) delta da raiz quadrada do erro quadrático médio ( $\left.\Delta_{\text {RQEM }}\right)$ : $\Delta$ RQEM $=$ Média $\left(\right.$ RQEM) N [2...6] - Média $(\text { RQEM })_{N[1]}$

Em que, $N$ [2..6] : 6 melhores redes com 2 a 6 neurônios na camada oculta; N [1] : 6 melhores redes com 1 neurônio na camada oculta.

Para testar possíveis diferenças estatísticas entre as alturas estimadas pelas redes e as alturas observadas para o conjunto de dados da validação, foram utilizados o teste t pareado ( $95 \%$ de probabilidade) e teste $\chi 2$ (99\% de probabilidade). Para ambos os testes, as seguintes hipóteses foram formuladas:

$\mathrm{H}_{0}$ : Não houve diferença significativa na estimativa da altura por meio da RNA;

$\mathrm{H}_{1}$ : Houve diferença significativa na estimativa da altura por meio da RNA.

Para ambos os testes, rejeitou-se $\mathrm{H}_{0}$ na ocasião da estatística-teste calculada apresentar-se maior ou igual à tabelada.

As estatísticas-teste foram calculadas por:

$$
\begin{gathered}
t_{\text {calculado }}=\frac{\overline{\Delta \mathrm{h}}}{\frac{S_{\Delta \mathrm{h}}}{\sqrt{n}}} \\
\chi_{\text {calculado }}^{2}=\sum_{i=1}^{n}\left[\left(h_{\mathrm{i}}-\widehat{h}_{\mathrm{l}}\right)^{2} / \widehat{h}_{\mathrm{1}}\right]
\end{gathered}
$$

Em que: $\overline{\Delta h}=$ médias das diferenças entre as alturas observadas e estimadas; $S_{\Delta \mathrm{h}}=$ desvio padrão das 
diferenças entre as alturas observadas e estimadas; $n$ : quantidade de árvores; $h_{\mathrm{i}}=$ altura observada; $\hat{h}=$ altura estimada.

As análises estatísticas foram realizadas no software $R$ (R CORE TEAM, 2020).

\section{RESULTADOS}

\section{Caracterização das árvores amostradas}

As estatísticas descritivas (Tabela 1) apresentam pequena variabilidade para as variáveis dendrométricas, refletindo a característica do povoamento florestal.

Tabela 1. Resumo das variáveis dendrométricas usadas no treinamento e validação das RNA.

\begin{tabular}{cccccc}
\hline Variável & Tipo & Mínimo & Média & Máximo & $\begin{array}{c}\text { Desvio- } \\
\text { Padrão }\end{array}$ \\
\hline d & \multirow{2}{*}{ Treino } & 15,8 & 19,1 & 22,1 & 1,3 \\
h & & 20,4 & 25,6 & 30,1 & 1,9 \\
\hline d & \multirow{2}{*}{ Validação } & 16,2 & 19,1 & 22,2 & 1,3 \\
h & & 19,7 & 25,8 & 30,5 & 1,8 \\
\hline
\end{tabular}

Determinação da quantidade de neurônios na camada oculta

Na Figura 2 pode-se observar a variação das estatísticas re RQEM de 100 redes, segundo o número de neurônios na camada oculta, sendo de 1 a 6 neurônios.

Utilizando-se apenas 1 neurônio na camada oculta, demonstra-se que as 100 redes tenderam a ter uma performance constante, com $r$ aproximadamente de 0,61 e o RQEM de 1,48 .

Utilizando-se 2 neurônios na camada oculta, observase que na rede 1 o ré $\approx 0,67$ e o $R Q E M \approx 1,38$, havendo uma variação significativa na performance até a rede 5 , sendo o r de 0,63 e o RQEM de 1,31, se estabilizando até a rede 50 e, novamente, havendo uma variação, em que na rede 100 o valor de ré $\approx 0,61$ e o $R Q E M \approx 1,47$.

Utilizando-se 3 neurônios na camada oculta, observase que da rede 1 a 10 o ré $\approx 0,68$ e o RQEM $\approx 1,37$ e, após a rede 10 , há uma variação de performance contínua, sendo o $r \approx 0,62$ e o $R Q E M \approx 1,47$ na rede número 100 . De acordo com o método de Fletcher-Gloss (SILVA et al., 2010), esta quantidade de neurônios na camada oculta corresponderia ao número ideal de neurônios.

Utilizando-se 4 neurônios na camada oculta, observa- se que na rede 1 o ré $\approx 0,70$ e o $R Q E M \approx 1,33$, havendo leve variação de performance até, aproximadamente, a rede 33 , onde o $r$ é $\approx 0,67$ e o $R Q E M \approx 1,37$. Após a rede 33 , ocorre variação maior no valor de $r$, sendo de $\approx 0,62$ e o RQEM de $\approx 1,47$ na rede 100 .

Utilizando-se 5 neurônios na camada oculta, nas primeiras redes o $r$ é $\approx 0,71$ e o $R Q E M \approx 1,31$, havendo variação leve na performance até $\mathrm{a}$, aproximadamente, rede 50 , onde o $r$ é $\approx 0,67$ e o $R Q E M \approx 1,37$, seguido de maior variação, onde na última rede, a de número 100 , o valor de $r$ é $\approx 0,61$ e o $R Q E M \approx 1,47$.

Utilizando-se 6 neurônios na camada oculta, nas primeiras redes o $r$ é $\approx 0,72$ e o $\operatorname{RQEM} \approx 1,27$, até, aproximadamente, a rede 65 , havendo pequena variação na performance, sendo o valor do $r$ de $\approx 0,67$ e o RQEM de $\approx 1,28$. Na sequência, houve maior variação, sendo o valor de $r$ de $\approx 0,61$ e $R Q E M$ de $\approx 1,47$ na rede 100 .

A Tabela 2 apresenta as seis melhores redes para cada número de neurônio na camada oculta e as estatísticas de qualidade $r$, RQEM, teste $t$ pareado e $\chi^{2}$ para cada rede, além de apresentar o $\Delta_{\mathrm{r}}$ e $\Delta_{\text {RQEM }}$ para cada configuração de neurônio na camada oculta. Observam-se os valores de $0,04,0,07,0,08,0,09,0,10$ para $\Delta_{r}$ no treino aplicando a equação 5, para 2, 3, 4, 5 e 6 neurônios na camada oculta, respectivamente; e para validação -0,05, 0,05, 0,08, 0,05 e 0,07 . Aplicando a equação 6 , encontrou-se o valor de $\Delta_{\text {RQEM }}$ no treino de $-0,07,-0,11,-0,13,-0,16,-0,17$ e para validação $0,08,0,08,0,11,0,08,0,12$ para $2,3,4,5$ e 6 neurônios na camada oculta, respectivamente.

\section{Análise gráfica dos resíduos}

Na Figura 3, observa-se o gráfico de resíduos em relação ao diâmetro para cada número de neurônios na camada oculta. Quando utilizado 1, 3 e 6 neurônios, os dados tenderam a ser mais homogêneos, distribuídos mais próximos a linha do 0 . Em relação a 2, 4 e 5 neurônios, os erros foram levemente maiores com o aumento do d.

\section{Performance de todas as redes na validação}

Observou-se a performance individual de cada rede na validação, com destaque para a rede 22 , que foi a melhor no treino com o valor de $r \approx 0,68$ e RQEM $\approx 1,37$ (Figura 4). É notável verificar outras redes que obtiveram valores superiores nas estatísticas de qualidade na validação, como no caso da rede 25,41 e 87. 
(a) 1 neurônio na camada oculta
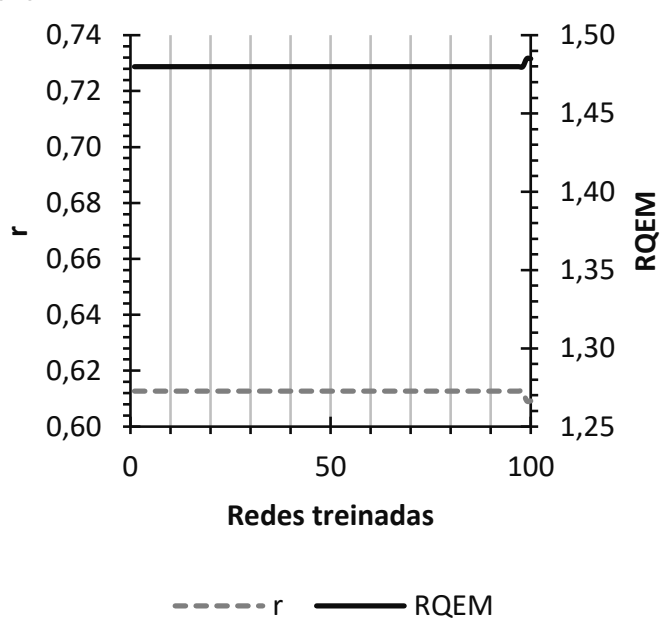

(c) 3 neurônios na camada oculta

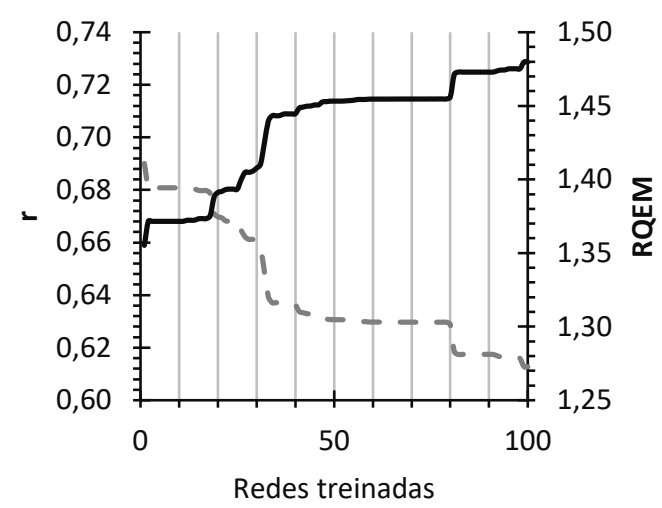

$--r \longrightarrow$ RQEM

(e) 5 neurônios na camada oculta

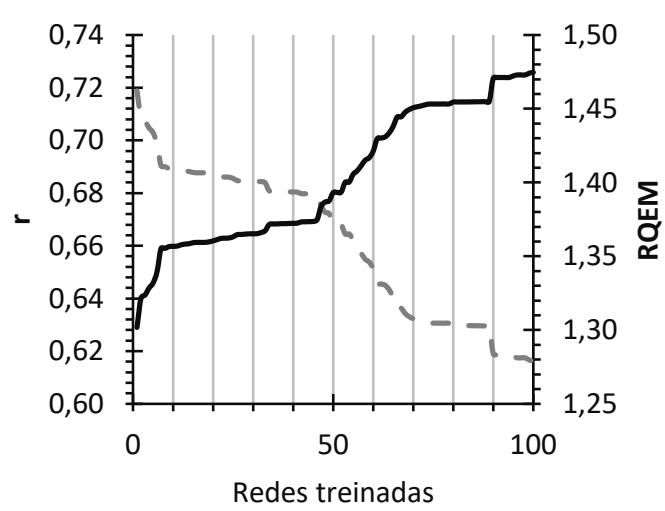

$--r \quad$ RQEM (b) 2 neurônios na camada oculta

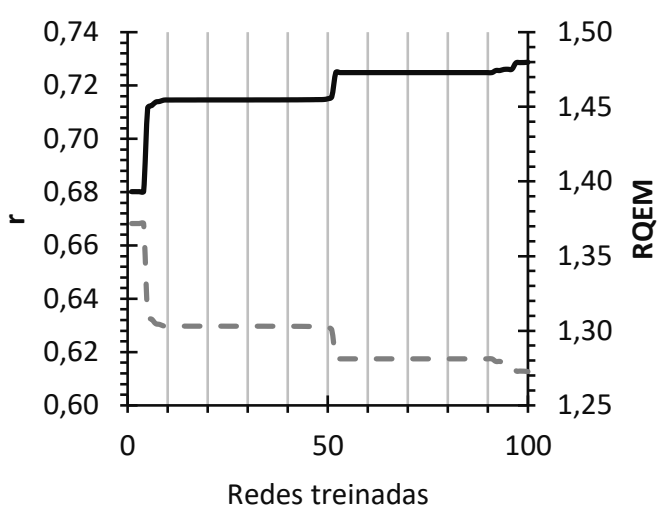

$--r \longrightarrow$ RQEM

(d) 4 neurônios na camada oculta



$--r \longrightarrow$ RQEM

(f) 6 neurônios na camada oculta

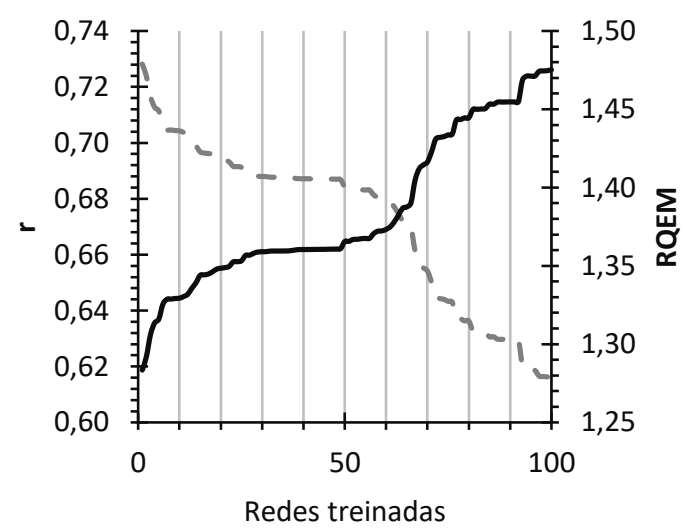

$---r \longrightarrow$ RQEM

Figura 2. Performance dos critérios estatísticos usados no treinamento e na seleção das seis melhores RNA para estimar a altura total de árvores de eucalipto em função do diâmetro a 1,30 m com configuração variando de um a seis neurônios da camada oculta. 
Tabela 2. Estatística de qualidade para validação e treino das seis melhores RNA para estimar a altura total de árvores de eucalipto em função do diâmetro a 1,30 m, com configuração variando de um a seis neurônios na camada oculta.

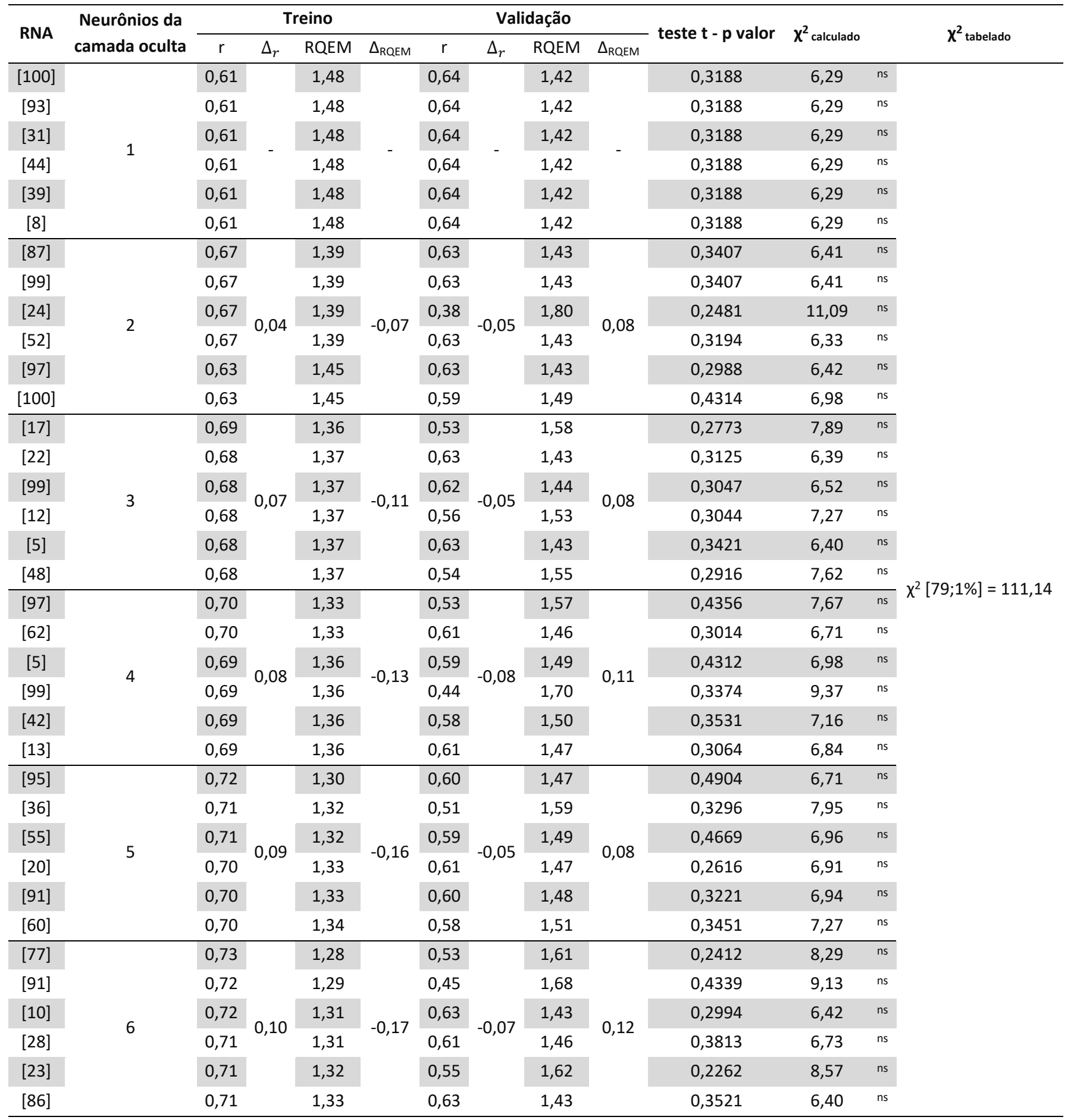

Em que: $r$ = coeficiente de correlação, $\Delta_{r}=$ Delta do coeficiente de correlação, RQEM = raiz quadrada do erro quadrático médio, $\Delta_{\mathrm{RQEM}}=$ delta da raiz quadrada do erro quadrático médio, $\chi^{2}=$ qui-quadrado. 
(a) RNA100 [d] - 1 neurônio na camada oculta

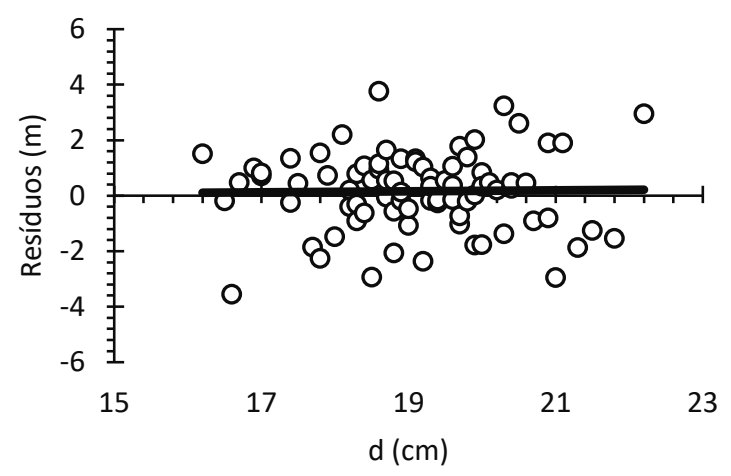

(c) RNA22 [d] - 3 neurônios na camada oculta



(e) RNA95 [d] - 5 neurônios na camada oculta

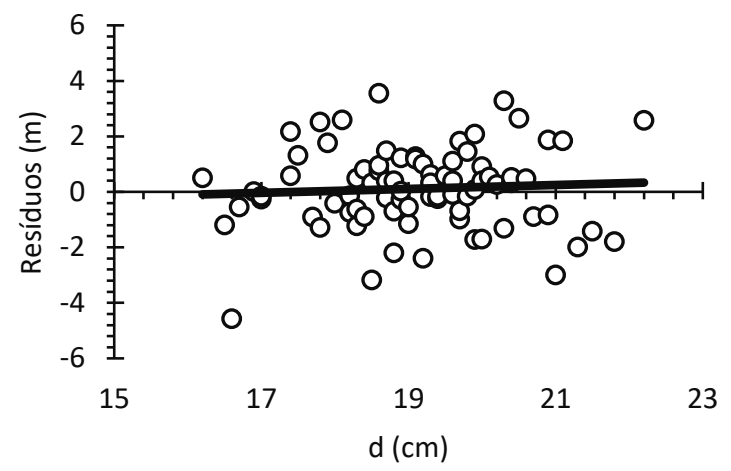

(b) RNA87 [d] - 2 neurônios na camada oculta

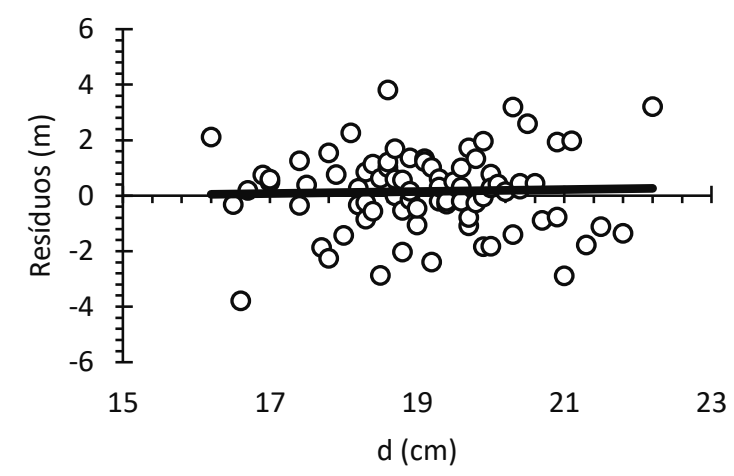

(d)

RNA62 [d] - 4 neurônios na camada oculta

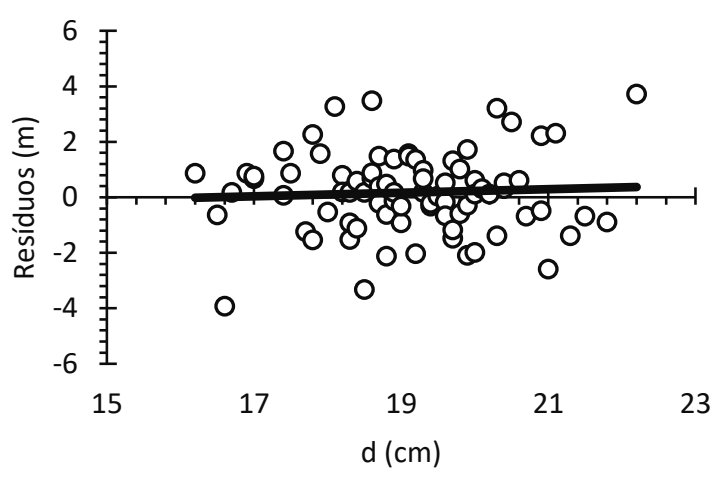

(f)

RNA10 [d] - 6 neurônios na camada oculta



Figura 3. Análise residual da validação das seis melhores RNA para estimar a altura total de árvores de eucalipto em função do diâmetro a 1,30 m, com configuração variando de um a seis neurônios na camada oculta. 
RNA22 [d] - 3 neurônios da camada oculta [Validação]



Figura 4. Performance da validação das 100 redes com 3 neurônios na cama oculta para estimar a altura total de árvores de eucalipto em função do diâmetro a 1,30 m. (Simbologia em vermelho: melhor rede treinada; Simbologia em azul: melhores redes na validação).

\section{DISCUSSÃO}

Com relação à performance dos critérios estatísticos para as diferentes arquiteturas (Figura 2), com 1 neurônio na camada oculta, todas as redes tiveram a mesma performance, já com 2 neurônios na camada oculta, a partir da terceira rede é notável o declínio de performance, evidenciado no gráfico pelas estatísticas de qualidade, ocorrendo underfitting (baixo ajuste). Com 3 neurônios, observou-se um melhor comportamento gráfico, visto que proximamente da terceira à vigésima rede ocorreu desempenho constante das redes. Com 4, 5 e 6 neurônios observou-se uma possível memorização dos dados de treinamento na validação, ocorrendo overfitting (super ajuste) devido ao excessivo número de neurônios utilizado na camada oculta (MARTINS et al., 2020).

Logo, faz-se extremamente necessário utilizar uma rede com o número ideal de neurônios na camada oculta permitindo, desta forma, que o modelo seja parcimonioso e não gere problemas como overfitting e underfitting (CUNHA NETO et al., 2019).

Existem várias equações para se determinar o número ideal de neurônios, com o método Fletcher-Gloss (SILVA et al., 2010) que, segundo sua expressão, o número ideal para o presente estudo seria de 3 neurônios na camada oculta. Diversos outros métodos podem ser utilizados para encontrar esse número, como os métodos detalhados no trabalho de Sheela e Deepa (2014).

$\mathrm{Na}$ Tabela 2, evidencia-se, segundo as estatísticas de qualidade $r$ e RQEM, a superioridade no treinamento das redes com maior número de neurônios na camada oculta. Entretanto, apesar do aumento do número de neurônios na camada oculta, a performance da rede tendeu a se estabilizar. $\mathrm{Na}$ validação, a performance não possuiu o mesmo comportamento comparado ao treinamento, segundo as estatísticas de qualidade, uma vez que mostrou nitidamente a superioridade das redes com menor número de neurônios na camada oculta, ressaltando a ocorrência de overfitting, ao se adotar número muito elevado de neurônios. Para modelos simples com uma única variável de entrada, como no presente trabalho, maior número de neurônios pode tornar a rede muito complexa e, consequentemente, ocasionar declínio em sua performance.

$\mathrm{Na}$ Figura 4, uma diferença de performance entre treino e validação é observada. A rede 22, a melhor durante o treinamento, não foi a melhor na validação segundo as estatísticas de qualidade, atenta-se que há outras redes com melhor desempenho. Cabe mencionar que a melhor rede no treinamento não necessariamente será também a melhor rede durante a validação. Assim, torna-se de extrema importante reservar uma parte dos dados que será destinado à validação.

Apesar da diferença no comportamento da performance de treino e validação, observa-se que com o uso de um número adequado de neurônios (em torno de 3) é possível obter resultados bastante satisfatórios de $r$ e RQEM para a validação, com valores bastante próximos aos do treinamento. Isso indica a capacidade de generalização do modelo gerado, o que permite sua aplicação em 
situações diferentes daquela em que foi gerado, para predições acuradas de altura da espécie.

O uso de dados não apenas da amostra em si, mas, também, dados de uma amostra externa para a validação é recomendado, possibilitando a verificação da performance das redes de maneira mais eficaz.

\section{CONCLUSÕES}

Para determinação da altura de eucalipto nas condições do presente estudo, os modelos preditivos gerados via rede neural MLP apresentaram bons treinos.

Uma estrutura de rede com 3 neurônios na camada oculta foi a ideal para geração de modelo, sem problemas de overfitting e underfitting.

Com 3 neurônios na camada oculta, a rede 22 obteve melhor performance no treino, porém, na validação, as redes 25, 41 e 87 obtiveram melhor performance, sendo, portanto, crucial a reserva de uma parte da amostra para validação dos dados. Além disso, quando possível, realizar a validação em dados externos à amostra para verificação da performance das redes.

Com o uso de um número adequado de neurônios, a modelagem da relação hipsométrica para a espécie apresentou boa capacidade de generalização.

\section{REFERÊNCIAS}

ALVARES, C.A. et al. Köppen's climate classification map for Brazil. Meteorologische Zeitschrift, v.22, p.711-728, 2013.

BRAGA, A.P. et al. Redes Neurais Artificiais: teoria e aplicações. Livros Técnicos e Científicos Editora S.A, 2000.

CAMPOS, B.P.F. et al. Predição da altura total de árvores em plantios de diferentes espécies por meio de redes neurais artificiais. Pesquisa Florestal Brasileira, v.36, n.88, p.375-385, 2017.

CUNHA NETO, E.M. et al. Estimativa do afilamento do fuste de indivíduos de eucalipto por meio de técnicas de inteligência artifical. BIOFIX Scientific Journal, v.4, n.2, p.166-171, 2019.

LACERDA, T.H.S. et al. Redes neurais artificiais para estimar o volume de árvores no cerrado. Cerne, v.23, n.4, p.483-491, 2017.

LEAL, F.A. et al. Redes neurais artificiais na estimativa de volume em um plantio de eucalipto em função de fotografias hemisféricas e número de árvores. Revista Brasileira de Biometria, v.33, n.2, p.233-249, 2015.

MARTINS, M.T. et al. Estimação da altura de plantios florestais de eucalipto por regressão e redes neurais artificiais. BIOFIX Scientific Journal, v.5, n.1, p.141-152, 2020.

MONTANO, R.A.N.R. et al. Artificial Intelligence Models to
Estimate Biomass of Tropical Forest Trees. Polibits, v.56, n.1, p.29-37, 2017.

NASIR, V. et al. Prediction of physical and mechanical properties of thermally modified wood based on color change evaluated by means of "group method of data handling" (GMDH) neural network. Holzforschung, v.73, n.4, p.381-392, 2019.

ÖZÇELIK, R. et al. Artificial Neural Network Models: An Alternative Approach for Reliable Aboveground Pine Tree Biomass Prediction. Forest Science, v.63, n.3, p.291-302, 2017.

$R$ CORE TEAM. R: A language and environment for statistical computin. R Foundation for Statistical Computing. Vienna, 2020. Disponível em: <https://www.R-project.org/>

SAKICI, O.E.; OZDEMIR, G. Stem taper estimations with artificial neural networks for mixed oriental beech and kazdaği fir stands in karabük region, Turkey. Cerne, v.24, n.4, p.439-451, 2018.

SATO, Y. et al. Artificial Neural Networks and Statistical Model. Japanese journal of applied statistics, v.24, n.2, p.77-88, 1995.

SCHIKOWSKI, A.B. et al. Estudo da forma do fuste utilizando redes neurais artificiais e funções de afilamento. Pesquisa Florestal Brasileira, v.35, n.82, p.119-127, 2015.

SCHMIDT, M. et al. A spatially explicit height-diameter model for Scots pine in Estonia. European Journal of Forest Research, v.130, n.2, p.303-315, 2011.

SHEELA, K.G.; DEEPA, S.N. Selection of number of hidden neurons in neural networks in renewable energy systems. Journal of Scientific and Industrial Research, v.73, n.10, p.686-688, 2014.

SILVA, I.N. et al. Redes Neurais Artificiais: Para Engenharia e Ciências Aplicadas. São Paulo: ARTLIBER, 2010.

TKÁČ, M.; VERNER, R. Artificial neural networks in business: Two decades of research. Applied Soft Computing Journal, v.38, p.788-804, 2016.

TUAN, N.T. et al. Height-diameter relationship for Pinus koraiensis in Mengjiagang Forest Farm of Northeast China using nonlinear regressions and artificial neural network models. Journal of Forest Science, v.65, n.4, p.134-143, 2019.

VENDRUSCOLO, D.G.S. et al. Estimativa Da altura de eucalipto por meio de regressão não linear e redes neurais artificiais. Revista Brasileira de Biometria, v.33, n.4, p.556-569, 2015.

VIEIRA, G.C. et al. Prognoses of diameter and height of trees of eucalyptus using artificial intelligence. Science of the Total Environment, v.619-620, p.1473-1481, 2018.

ZHOU, R. et al. Estimation of DBH at Forest Stand Level Based on Multi-Parameters and Generalized Regression Neural Network. Forests, v.10, n.9, p.778-796, 2019.

Recebido em 28-01-2020 Aceito em 11-03-2020 